\section{IN BRIEF}

\section{$\Rightarrow$ OSTEOARTHRITIS}

\section{Fat mass but not muscle mass linked to OA risk}

The risk of incident radiographic knee osteoarthritis (OA) over 5 years differed depending on an individual's body composition (as determined by whole body dual energy $\mathrm{x}$-ray) in a longitudinal cohort $(n=1,653)$. Sarcopenia was not associated with an increased risk of knee OA in men (relative risk (RR) 0.66, $95 \% \mathrm{Cl} 0.34-1.30$ ) or in women (RR 0.96, 95\% Cl 0.62-1.49). By contrast, obesity was associated with an increased risk of knee OA in men (RR 1.73, 95\% Cl 1.08-2.78) and both obesity and sarcopenic obesity were associated with an increased risk of knee OA in women (obesity RR 2.29, 95\% Cl 1.64-3.20; sarcopenic obesity RR 1.91, 95\% Cl 1.17-3.11).

ORIGINAL ARTICLE Misra, D. et al. Risk of knee OA with obesity, sarcopenic obesity and sarcopenia. Arthritis Rheumatol. https://doi.org/10.1002/art.40692 (2018)

\section{$\Rightarrow$ THERAPY}

\section{Intensive therapy is beneficial in early arthritis}

Remission could be induced and sustained in patients with early inflammatory arthritis (defined as synovitis of 12 weeks duration in at least two joints; $n=90$ ) following 1 year of treatment with infliximab and methotrexate (MTX) in a randomized placebocontrolled trial. Patients receiving infliximab plus MTX were more likely to achieve remission at 54 weeks than patients receiving MTX alone or those receiving placebo (34\% achieved remission on infliximab plus MTX versus $0 \%$ on placebo; $P<0.05)$. Three-quarters of patients who achieved remission on infliximab plus MTX maintained remission throughout the following year with no therapy.

ORIGINAL ARTICLE Stamm, T. A. et al. Induction of sustained remission in early inflammatory arthritis with the combination of infliximab plus methotrexate: the DINORA trial. Arthritis Res. Ther. 20, 174 (2018)

\section{$\Rightarrow$ RHEUMATOID ARTHRITIS}

\section{Anti-TNF therapy not linked to cancer recurrence}

Patients with rheumatoid arthritis (RA) and a history of cancer had a similar risk of cancer recurrence regardless of whether or not they received anti-TNF therapy in a Swedish populationbased cohort study. Of the 467 patients with RA who started anti-TNF therapy a mean of 7.9 years after cancer diagnosis, $9 \%$ had cancer recurrence (mean follow-up 5.3 years) compared with $7.2 \%$ of matched patients with RA and a history of cancer who did not receive any form of biologic therapy $(n=2,164$; mean follow-up 4.3 years). This difference was not statistically significant (HR 1.06, 95\% Cl 0.73-1.54).

ORIGINAL ARTICLE Raaschou, P. et al. Tumor necrosis factor inhibitors and cancer recurrence in Swedish patients with rheumatoid arthritis: A nationwide population-based cohort study. Ann. Intern. Med. https://doi.org/10.7326/M17-2812 (2018)

\section{SYSTEMIC SCLEROSIS}

\section{Serum biomarkers for diagnosing PAH}

Serum concentrations of midkine (a growth factor) and follistatin-related protein 3 (FSTL3) could discriminate between patients with limited cutaneous systemic sclerosis (lcSSc) who did and did not have pulmonary arterial hypertension (PAH) in a discovery cohort of 29 patients. An increased serum concentration of both midkine and FSTL3 (as measured by enzyme-linked immunosorbent assay) was validated as a biomarker for PAH in two independent cohorts of patients with lcSSc $(n=35, n=29)$, in which it had a sensitivity of $76 \%$ and $91 \%$, respectively, and a specificity of $76 \%$ and $81 \%$, respectively.

ORIGINAL ARTICLE Rice, L. M. et al. Serum biomarker for diagnostic evaluation of pulmonary arterial hypertension in systemic sclerosis. Arthritis Res. Ther. 20, 185 (2018)

\title{
Distinguishing NET subtypes
}

Researchers have developed an approach for discriminating between neutrophil extracellular traps (NETs) released by nicotinamide adenine dinucleotide phosphate (NADPH) oxidase (NOX)-dependent and NOX-independent mechanisms. "We discovered that distinct disease groups have mainly either NOXdependent or NOX-independent NETs," explains Johan van der Vlag, corresponding author of the new study.

As a potential source of autoantigens, NETs are implicated in the pathogenesis of a number of autoimmune diseases. These structures, which are composed of DNA with histones and granule enzymes (such as myeloperoxidase (MPO) and neutrophil elastase), are released by neutrophils in response to various stimuli.

NOX-dependent NET formation involves the assembly of NOX and subsequent generation of reactive oxygen species, culminating in lytic release of NETs, whereas NOX-independent NETs are released through blebbing of the nuclear envelope. Current methods for quantifying NET formation in vivo measure MPO and DNA, but do not distinguish between NETs produced by NOX-dependent and NOX-independent pathways.

"We discovered that the $\mathrm{N}$-terminal histone tails were cleaved off in NOX-dependent NETs by neutrophil elastase, but not in NOX-independent NETs," remarks van der Vlag. Given these findings, the researchers developed sandwich ELISAs that employed antibodies against either the $\mathrm{N}$-terminal tails or $\mathrm{C}$-terminal regions of histones as the capturing antibodies, and anti-MPO antibodies as the detecting antibodies. ELISAs employing antibodies against the $\mathrm{N}$-terminal histone tails could detect NOX-independent NETs but not NOX-dependent NETs, whereas ELISAs that employed antibodies against the histone $\mathrm{C}$-terminal region could detect both NOX-dependent and NOX-independent NETs equally.

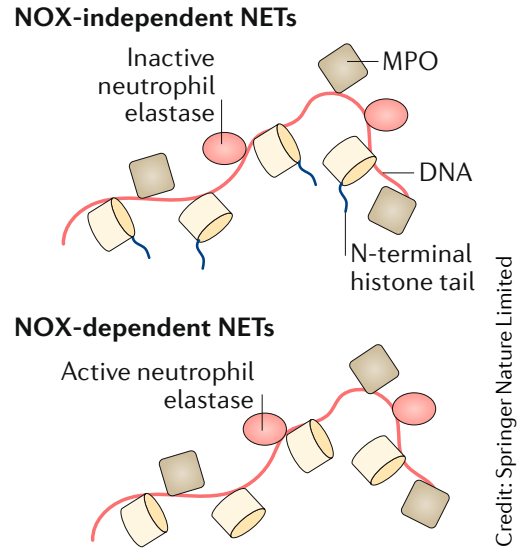

To ascertain the in vivo relevance of NOX-dependent and NOX-independent NET production, they measured levels of NETs in the plasma of patients with various NET-associated diseases. In patients who were positive for MPO-DNA complexes, most of those with psoriatic arthritis tested negative for NOX-independent NETs, whereas the majority of patients with either rheumatoid arthritis or systemic lupus erythematosus tested positive.

In vitro, NOX-independent NETs had greater immunostimulatory effects on endothelial cells than NOX-dependent NETs and induced the expression of various adhesion markers and pro-inflammatory cytokines.

Going forward, van der Vlag's group plan to apply this approach to measure NOX-dependent and NOX-independent NETs in large cohorts of patients with rheumatic diseases, develop strategies that specifically interfere with NOX-dependent or NOX-independent NET formation, and elucidate the mechanisms underlying NET-mediated activation of endothelial cells.

Jessica McHugh

ORIGINAL ARTICLE Pieterse, E. et al. Cleaved $\mathrm{N}$-terminal histone tails distinguish between NADPH oxidase (NOX)-dependent and NOXindependent pathways of neutrophil extracellular trap formation. Ann. Rheum. Dis. https://doi.org/ 10.1136/annrheumdis-2018-213223 (2018) 